Case Report

\title{
Vestibular nerve compression: role of auditory brainstem response and cervical vestibular evoked myogenic potentials
}

\author{
Deepa A. Valame*, Geeta B. Gore
}

\begin{abstract}
Department of Audiology and Speech Therapy, T.N. Medical College and B.Y.L. Nair Hospital, Mumbai, Maharashtra, India
\end{abstract}

Received: 07 April 2017

Accepted: 19 May 2017

\section{*Correspondence:}

Mrs. Deepa A. Valame,

E-mail: deepa_valame@yahoo.co.in

Copyright: ( ) the author(s), publisher and licensee Medip Academy. This is an open-access article distributed under the terms of the Creative Commons Attribution Non-Commercial License, which permits unrestricted non-commercial use, distribution, and reproduction in any medium, provided the original work is properly cited.

\begin{abstract}
The objective of the study was to evaluate the role of ABR and cVEMP in the diagnosis of vestibular compression syndrome (VCS) and to study the association of test results with the MRI findings. This is a case-report of four patients with VCS using case-study approach. Four patients with varying degrees of indentation of vestibulo-cochlear nerve by the anterior inferior cerebellar artery (AICA) loops were studied. Episodic rotatory vertigo was reported by three cases and two cases complained of tinnitus but the characteristic low-frequency 'type-writer' type of tinnitus was seen in only one. All cases showed evidence of retrocochlear pathology on ABR although two had normal peripheral hearing status. The cVEMP abnormalities noted were absence of cVEMP and reduced amplitude of cVEMP as compared to instrument-specific age-matched norms; only one case with no indentation of vestibulocochlear nerve had normal cVEMP tracings. Presence of AICA loops on the MRI by itself need not necessarily indicate vestibular compression syndrome. However when MRI excludes any other pathology in cases with symptoms such as unilateral sensorineural hearing loss, tinnitus, vertigo; vestibular compression could be the etiology. The likelihood of abnormal test findings is greater when the loop causes indentation of the nerve.
\end{abstract}

Keywords: Vestibular Compression syndrome, cVEMP, AICA loops, ABR

\section{INTRODUCTION}

Vertigo is a commonly encountered symptom in a neuroaudiological set-up and may be seen as a result of myriad of conditions-peripheral or central. One such condition characterized by repeated episodes of rotatory vertigo, tinnitus and hearing loss due to irritability of the vestibular nerve is Vestibular Compression syndrome (VCS). This condition was first recognized by Janetta, Moller and Moller who reported of patients with tinnitus, sensorineural (SN) hearing loss, spinning vertigo associated with compression of the vestibular nerve by loop of anterior inferior cerebellar artery (AICA). ${ }^{1}$ The patients showed absent stapedial reflexes and delayed interpeak latency interval (IPL) for wave III-I on the auditory brainstem response (ABR). They had previously reported of such microvascular compression of trigeminal nerve and the facial nerve by vascular loops leading to hemifacial spasms and trigeminal neuralgia.

This clinical entity, however, is controversial with respect to its existence, diagnosis as well as its management. The various aspects that are in controversy are:

1. The pathophysiology of vascular compression of cranial nerves is better explained for motor nerves than sensory nerves.

2. MRI findings of vascular loops indenting the eighth cranial nerve have been reported in many asymptomatic patients leading to the speculation that whether the symptoms are truly as a result of 
microvascular compression or is the compression an incidental finding with the auditory symptoms due to another unknown/undiagnosed cause.

3. The symptoms of this condition (disabling vertigo, tinnitus, hearing loss, nausea etc) are non-specific and could be seen in variety of conditions.

4. There are no gold-standard diagnostic tests although MRI and MRA scans are most often cited in literature. Actually, their main role is to exclude other causes such as tumors rather than identify vascular loops.

5. In management, if one believes that the vestibular nerve irritation is truly due to vascular loop indentation, then a surgical approach to management is logical whereas medical management would be preferred if the symptoms are due to other causes such as concomitant Meniere's disease.

Mc Dermott et al reported that the symptoms of VCS correlate with the extension of the vascular loop into the internal auditory canal (IAC). ${ }^{2}$ He classified AICA loops as:

Type I: Loop in the cerebello-pontine angle (CPA) but not extending into the IAC.

Type II: Loop extending into IAC and covering less than $50 \%$ of the length of IAC.

Type III: Loop extending into IAC and covering more than $50 \%$ of the length of IAC.

They found type III loops to be significantly associated with unilateral SN loss although he reported no association of tinnitus with type of loop. This finding has been refuted in other studies cited in literature. One study reported correlation of type I and type II small AICA loops with unexplained tinnitus. ${ }^{3}$ Their results were in agreement with the hypothesis that compression of the eighth cranial nerve by AICA loops at the root entry zone of the nerve led to impaired blood circulation and tinnitus. However they did not find any association of the type of loop with hearing loss.

Another classification system was used by Gorrie, Warren, Garza, Shelton and Wiggins where they classified the vascular loops based on the contact made by the loops with the cranial nerves: loops that were not in contact with the nerve, loops running adjacent to the nerve but not displacing it, loops displacing the vestibulocochlear nerve, and loops running between the facial and the vestibulo-cochlear nerve. ${ }^{4}$ They reported no association of tinnitus or hearing loss with the type of AICA loop as per the Mc Dermott classification but when their new classification system was used, they found a statistically significant association between loops that ran between the facial and vestibulo-cochlear nerve with hearing loss, as well as with tinnitus.
Audiological tests used in the diagnostic work-up of VCS have been mainly pure tone audiometry (PTA), and auditory brainstem response (ABR). PTA findings in such cases are highly variable although Moller reported the presence of mid-frequency hearing loss. ${ }^{5}$ Their study also showed ABR diagnostically useful with presence of interpeak latency (IPL) delay of II-I and III-I in their patients. Recently, Singh, Singh, Usha and Akshay used a comprehensive audiovestibular test battery on two patients with VCS whose MRI showed presence of vascular loop around the seventh and eighth cranial nerves. ${ }^{6}$ Their patients showed no abnormalities on PTA, ABR and cervical vestibular evoked myogenic potentials (cVEMP), but revealed unilateral caloric weakness, spontaneous nystagmus and positive findings on Dix-Hall pike tests.

Thus, although there are many studies on VCS, most of them in form of case -reports; there is no consensus among them about whether the auditory symptoms result from the compression, what are the symptoms and their association with type of loop. Further, most recent studies have used MRI/MRA and ABR as diagnostic tools and only few have explored the role of cervical Vestibular evoked myogenic potentials (cVEMP) in the evaluation of this condition. Since the cVEMP evaluates the inferior vestibular branch and the ABR evaluates the cochlear branch of eighth nerve, more studies using these tests may add to our understanding of this clinical entity. Casereports of such patients will surely enhance our present understanding and guide us in future management of these patients.

The present case-report presents four patients with AICA loops with different degrees of indentation of the eighth cranial nerve, discusses their symptoms as well as their findings on PTA, ABR and CVEMP and attempts to associate these results with MRI findings.

\section{CASE REPORT}

\section{Case A}

M/68 years reported with complaint of decreased hearing in the right ear since three to four years, episodic spinning vertigo, sensation of aural fullness and nausea. He had no complaint of tinnitus or headache. The audiological work-up aimed to determine the site of lesion as cochlear vs. retrocochlear and included PTA, acoustic reflex testing, ABR and cVEMP. On PTA, case A showed unilateral moderately severe sensorineural hearing loss with left ear hearing sensitivity within normal limits except mild hearing loss at high frequencies. Acoustic reflexes were absent ipsilaterally as well as contralaterally at all frequencies tested whenever the stimulus was presented to the right ear.

$\mathrm{ABR}$ and cVEMP were carried out using intelligent hearing system (HIS) Smart EP (Florida, USA) using 0.1 msec clicks and $500 \mathrm{~Hz}$ tone bursts respectively. For 
$\mathrm{ABR}$, the clicks were presented at repetition rates of 9.3/sec, $19.3 / \mathrm{sec}$ and $72 / \mathrm{sec}$ using alternate polarity. Response was obtained in a dual-channel recording with the active electrode placed at the vertex, the reference electrode placed at the mastoid and ground at forehead. The response was band-pass filtered from 100-3000 Hz and amplified $100 \mathrm{k}$ times. At all the repetition rates, the ABR wave morphology and replicability was fair. Right ear showed delayed latency of waves III and V resulting in delayed IPL III-I: $2.3 \mathrm{msec}$ and $2.8 \mathrm{msec}$ at $19.3 / \mathrm{sec}$ and $72 / \mathrm{sec}$ respectively. This led to delayed V-I IPL too. Left ear revealed normal latency of early peaks with marginally delayed wave $\mathrm{V}$ thus prolonging IPL V-III (2.23 msec and $2.3 \mathrm{msec}$ respectively at $19.3 / \mathrm{sec}$ and $72 / \mathrm{sec})$. These findings raised the suspicion of retrocochlear lesion in the right ear with mild mass effect on the opposite ear (Figure 1).

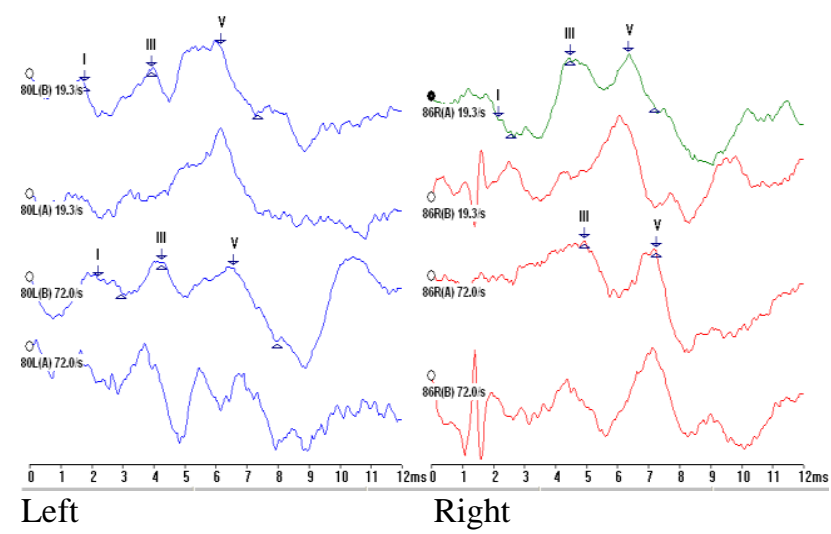

Figure 1: ABR in Case A.

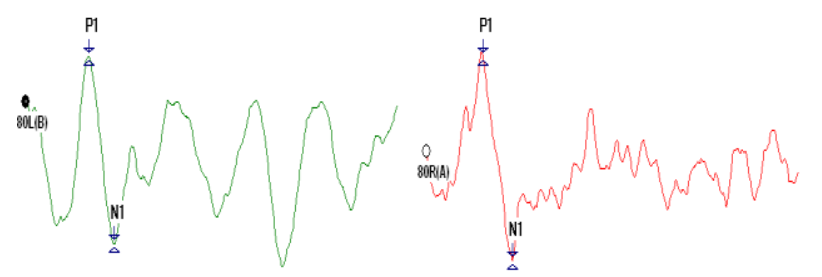

amplitude was reduced by almost $50 \%$ as compared to the normative value and with that of the left ear (Figure 2).

The client underwent MRI that revealed AICA loop indenting the seventh and eighth cranial nerve root entry zone on the right side. No other lesion/abnormality was seen on the MRI.

\section{Case B}

F/56 years reported with a complaint of decreased hearing in the left ear following stay in the intensive care unit for IHD six months back, episodic vertigo which was aggravated by postural changes and low pitched tinnitus. Audiogram revealed normal hearing sensitivity with a dip at $4000 \mathrm{~Hz}$ in the right ear and moderately severe sensorineural hearing loss in the left ear. Word recognition scores were $68 \%$ and $16 \%$ respectively for the right and left ears at most comfortable loudness level (MCL). Immittance testing revealed B type tympanogram, normal ear canal volume and absent ipsilateral and contralateral acoustic reflexes with probe in right ear indicative of middle ear effusion in the right ear. Left ear showed A type tympanogram with absent reflexes consistent with possibility of retrocochlear involvement.

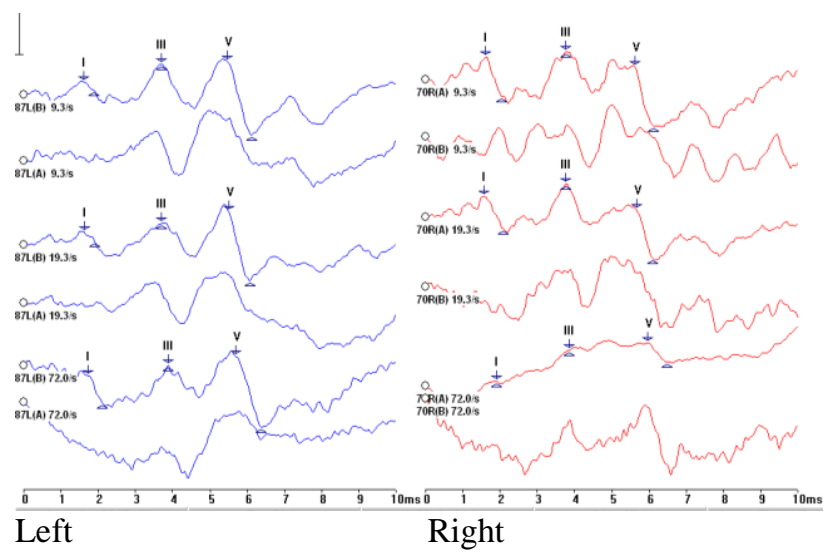

Figure 3: ABR in case $B$.

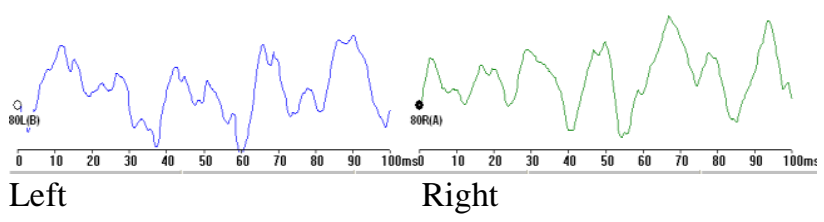

Figure 4: cVEMP in case $B$.

ABR and cVEMP were recorded on IHS smart EP using the same parameters as stated for case A. The ABR was characterized by good wave morphology and replicability

bilaterally with absolute latencies of all waves, IPL and
cVEMP was obtained monaurally from contracted sternocleidomastoid (SCM) muscle by asking the client to turn his head to the side opposite to the ear stimulated. Single -channel recording of response was done with sternoclavicular notch serving as reference electrode site and forehead as ground. The response was amplified $5 \mathrm{~K}$ times and band- pass filtered from $10-1000 \mathrm{~Hz}$. Morphology of response was poor bilaterally. Latencies of P1 and N1 were within normal limits. Amplitude of cVEMP was marginally reduced on left side as compared to instrument-specific age-matched norms. Right ear 
inter-aural latency difference (ILD) values within normal limits for both ears. cVEMP testing showed bilaterally no identifiable response (Figures 3 and 4).

Case B's MRI revealed a gliotic area in the right precentral region suggestive of old ischemic pathology. A prominent vessel, most probably AICA was seen to course along with the left seventh and eighth nerve complex but no neurovascular conflict was detected.

\section{Case C}

M/50 years reported with tingling sensation on right side of face, trigeminal neuralgia, he presented no ear-related complaints and was being evaluated by the neurologist for differential diagnosis of multiple sclerosis and vascular compression of trigeminal nerve. Interestingly, his MRI revealed a T2/flair hyper-intense area in the right postero-lateral pons and the right middle cerebellar peduncle suggestive of demyelination in trigeminal tract. An incidental finding was: Right SCA and branch of AICA passing antero-superior to the right trigeminal nerve without indentation/compression of nerve. There was a presence of AICA loop coursing around bilateral VII-VIII nerve complexes without evidence of indentation/compression of the nerves.

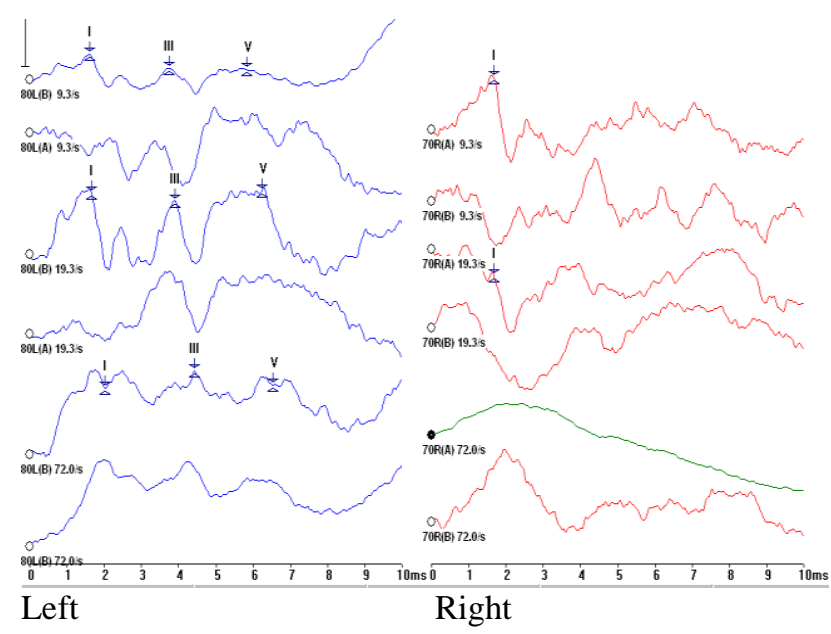

Figure 5: ABR in case $C$.

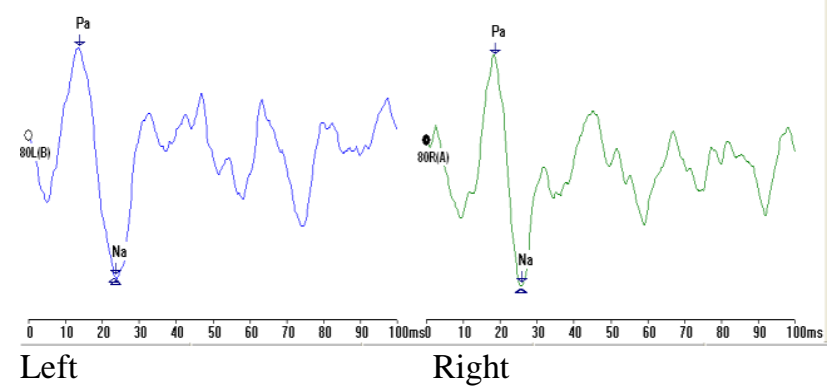

Figure 6: cVEMP in case $\mathrm{C}$.
Due to this finding, case $\mathrm{C}$ was taken up for audiological test battery. His audiogram revealed bilaterally normal hearing sensitivity. However Immittance revealed bilateral A type tympanograms with acoustic reflex absent when the stimuli were presented to the right ear. Left ear reflexes were elicited at normal hearing levels. Client was therefore taken-up for ABR and cVEMP testing. ABR was highly degraded in the right ear wherein no wave peaks could be identified except wave I at all repetition rates. Left ear showed good wave replicability with marginally delayed IPL. cVEMP response was within normal limits in the left ear ( latency of P1- $13.8 \mathrm{msec}$ and latency of N1-23.6 msec). Right ear cVEMP showed delayed latencies of P1 and N1 (18.6 msec and $25.8 \mathrm{msec}$ respectively) although amplitude was within one standard deviation of the instrumentspecific age-matched normative value (Figures 5 and 6).

\section{Case D}

$\mathrm{M} / 38$ years reported with decreased hearing in right ear, continuous high-pitched tinnitus in right ear, aural fullness and fast spinning episodic vertigo aggravated by positional changes since three years. His audiogram revealed bilateral hearing sensitivity within normal limits but Immittance revealed bilateral A type tympanograms with absence of acoustic reflex on the right side.

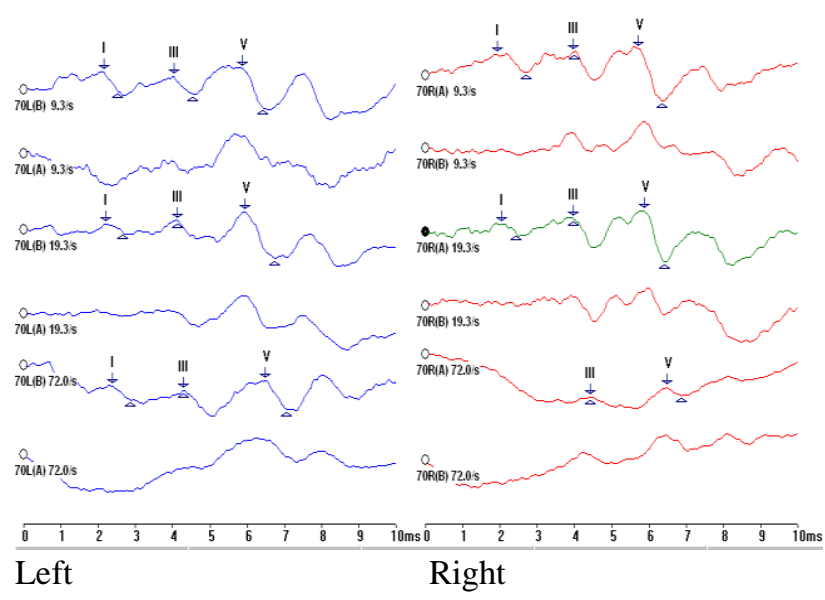

Figure 7: ABR in case $D$.

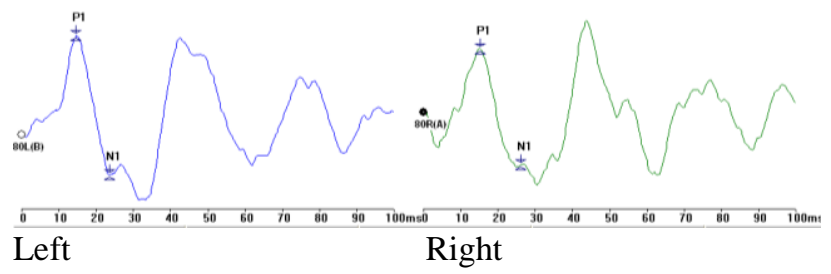

Figure 8: cVEMP in case D.

ABR showed that wave morphology and replicability was good bilaterally with absolute latencies, IPL for both ears 
and ILD within normal limits. cVEMP findings reveal normal values of latency of P1 and N1 with amplitude within normal limits (Figures 7 and 8). His MRI revealed a type I AICA loop abutting seventh and eighth cranial nerves with no compression.

\section{DISCUSSION}

AICA loops can cause compression of the vestibulocochlear nerve leading to audio-vestibular symptoms. Compression of the vascular loop can result due to disturbance in the perfusion of cochlea and vestibule or the compression may lead to nerve irritability and cause demyelination of the nerve at the root entry zone (REZ). ${ }^{7,8}$

Contrary to this, Schwaber and Whetsell suggested that association of vascular loops and cranial nerve symptoms were purely by chance, rather the symptoms were caused by a diffuse neuritis. ${ }^{9}$ They doubted whether contact alone would result in symptoms; rather, an episode of vestibular neuritis leading to axonal loss and swelling was deemed essential to cause the syndrome.

Of the four cases presented in this case-report, case A had AICA loop that caused indentation and compression of both seventh and eighth cranial nerves- most probably type III loop; B and C had loops that coursed in the IAC and around the cases seventh-eigth nerve complex (probably type II loops) and case D presented with a type I loop around abutting the seventh and eighth cranial nerves with no compression. Episodic rotatory vertigo was reported by all cases except case $C$ and the vertigo was aggravated by positional changes. Cases B and D complained of tinnitus but the characteristic lowfrequency 'type-writer' type of tinnitus was reported by case B whereas case D reported high pitched tinnitus. Case A with maximum indentation of the eighth nerve and a clear retrocochlear lesion did not complain of tinnitus. This was in agreement with findings of Yoo et al who showed that small sized AICA loops, mainly type I and II showed higher association with tinnitus as compared to type III loops. ${ }^{3}$ They concluded that small loops compressed the eighth nerve at the REZ and the impaired blood flow in these loops led to tinnitus.

Moderately severe sensorineural hearing loss in the affected ear was seen in cases A and B while the other two had no significant hearing loss. Both cases A and B with hearing loss appeared to have a retrocochlear type of hearing loss/ pathology based on the gestalt of their audiological test battery and cVEMP. Cases C and D too appeared to have retro-cochlear involvement despite normal peripheral hearing status. Although few studies in literature fail to clearly demonstrate association of AICA loop with presence of hearing loss, a meta-analytic study by Chadda and Weiner reported that patients with unilateral hearing loss were twice as likely to have AICA loops compressing the eighth nerve in their symptomatic ears. $^{6,10,11}$ In that case, the hearing loss must be retrocochlear in nature as demonstrated on audiological tests. In the present study, all patients showed evidence of retrocochlear involvement as seen in their absence of stapedial reflexes in the affected side (all cases), poor WRS (case A and B), delayed III-I IPL on ABR (cases A, $\mathrm{B}$ and $\mathrm{C}$ ) and abnormality of cVEMP ( cases A, B and C). Absence of stapedial reflex in the affected ear was consistent finding seen in all cases and thus stapedial reflex testing with $\mathrm{ABR}$ are recommended in the audiological battery of patients suspected of VSC.

We came across only one previously published study that used cVEMP in the test battery to evaluate these cases ${ }^{[6]}$ however this case report reported no significant abnormality on cVEMP. This could probably be due to the fact that their cases did not show clear impingement and compression of the eighth nerve by the loops. Case D of the present study with similar MRI findings also shows normal ABR and cVEMP response while the other cases in the present study show ABR and cVEMP affected on the affected side. The cVEMP abnormalities noted were absence of cVEMP and reduced amplitude of cVEMP as compared to instrument-specific age-matched norms. Case A showed mild effects even on the opposite side which had raised our suspicion of a space occupying lesion like vestibular schwannoma but MRI showed no abnormality other than a prominent vascular loop indenting the eighth nerve.

In the cases presented here, absence of cVEMP in the unaffected ear of case $B$ could be attributed to the presence of middle ear effusion in that ear which could preclude the recording of cVEMP response. Case $\mathrm{C}$ was the only one where the MRI raised suspicion of other pathology to explain the symptoms. His MRI also revealed a T2/ flair hyper intense area in right posterolateral pons and right middle cerebellar peduncle that appeared bright on diffusion. The radiologist thought it to be representing demyelination in the region of the trigeminal nucleus and hence the differential diagnosis in this case was between vascular compression syndrome and multiple sclerosis. Presence of tingling sensation on right side of face and trigeminal neuralgia favoured compression of trigeminal nerve as the MRI did show right SCA and branch of AICA passing antero-superior to the trigeminal nerve without indentation. Similar loop coursing around the seventh-eighth nerve complex could therefore be thought of causing the delayed latencies on the ABR. However absence of hearing loss, tinnitus and vertigo makes this possibility very unlikely. Rather, the delay in the latency of cVEMP waves on the right ear and complete absence of waves on ABR (except wave I) on the right side leads us to consider demyelinating pathology such as multiple sclerosis more likely.

Thus, presence of AICA loops on the MRI by itself need not necessarily indicate vestibular compression syndrome. The AICA follows a highly variable course and is frequently seen in contact with the $\mathrm{VCN}$ in asymptomatic ears as well. ${ }^{12}$ However when the 
MRI/MRA excludes any other pathology in cases with symptoms such as unilateral sensorineural hearing loss, tinnitus, vertigo; vestibular compression could be the aetiology. The likelihood of abnormalities on audiological test battery and symptoms is more when the vascular loop causes indentation/compression of the nerve thereby leading to hyperactivity of the nerve as compared to when there is presence of loop coursing around the eighth nerve without compression. To this end, the present study finds the classification of loops by Gorrie et al to be more clinically useful than the Mc Dermott anatomical classification. An audio-vestibular test-battery of PTA, stapedial reflex testing, ABR and cVEMP is recommended in such cases to ascertain the presence of retrocochlear hearing loss. On the other hand, when a patient reports with symptoms of unilateral sensorineural hearing loss, tinnitus and vertigo and audiovestibular tests show evidence of RCP, the possibility of VCS must be kept in mind.

\section{Funding: No funding sources}

Conflict of interest: None declared

Ethical approval: Not required

\section{REFERENCES}

1. Janetta PJ, Moller MB, Mollar AR. Disabling positional vertigo. N Eng J Med. 1984;310:1700-5.

2. McDermott A, Dutt S, Irving R, Pahor A, Chavda S. Anterior inferior cerebellar artery syndrome:fact or fiction? Clin Otolaryngol. 2003;28:75-80.

3. Yoo HS, Lee DW, Min HJ, Lee SH, Park CW. The Association of Anterior Inferior Cerebellar Artery in Internal Auditory Canal with Tinnitus and Hearing Loss. Korean J Audiol. 2011;15:67-71.

4. Gorrie A, Warren FM, Garza AN, Shelton C, Wiggins RH. Is there a correlation between vascular loops in the cerebellopontine angle and unexplained unilateral hearing loss? Otol Neurotol. 2010;31(1):48-52.

5. Moller AR. Vascular compression of cranial nerves:II:pathophysiology. Neurol Res. 1999;21:439-43.

6. Singh NK, Singh P, Usha M, Akshay M. Audiovestibular findings in Vestibular Paroxysmia. Indian J Otol. 2013;19:82-4.

7. Applebaum EL, Valvassori GE. Auditory and vestibular system. Findings in patients with vascular loops in the internal auditory canal. Ann Otol Rhinol Laryngol. 1984;92(112):63-70.

8. MtCabe BF, Gantz BJ. Vascular loop as a cause of incapacitating dizziness. Am J Otol. 1989;10:11720.

9. Schwaber MK, Whetsell WO. Cochleovestibular compression syndrome. II. Vestibular nerve histopathology and theory of pathophysiology. Laryngoscope. 1992;102:1030-6.

10. Clift JM, Wong RD, Carney GM, Stavinoha RC, Boyev KP. Radiographic analysis of cochlear nerve vascular compression. Annals Otol Rhinol Laryngol. 2009;118(5):391-6.

11. Chadha NK, Weiner GM. Vascular loops causing otological symptoms:a systematic review and metaanalysis. Clinical Otolaryngol. 2008;33:5-11.

12. Makins AE, Nikolopoulos TP, Ludman C, O'Donoghue GM. Is there a correlation between vascular loops and unilateral auditory symptoms? Laryngoscope. 1998;108:1739-42.

Cite this article as: Valame DA, Gore GB. Vestibular nerve compression: role of auditory brainstem response and cervical vestibular evoked myogenic potentials. Int J Otorhinolaryngol Head Neck Surg 2017:3:749-54. 